\title{
The transformation of hormonal stress responses throughout puberty and adolescence
}

\author{
Allison R Foilb, Patina Lui and Russell D Romeo \\ Department of Psychology and Neuroscience and Behavior Program, Barnard College of Columbia University, Milbank Hall, 3009 Broadway, New York, \\ New York 10027, USA \\ (Correspondence should be addressed to R D Romeo; Email: rromeo@barnard.edu)
}

\begin{abstract}
Prepubertal rats display heightened hormonal stress reactivity compared with adults in that levels of ACTH and corticosterone take twice as long (i.e. $40-60 \mathrm{~min}$ ) to return to baseline following an acute stressor. Despite this substantial change in stress responsiveness, and the critical nature of the adolescence period of development, the maturation of the hormonal stress response from the time of pubertal onset to adulthood has not been thoroughly investigated. To examine this, we measured ACTH, corticosterone, and testosterone in $30-, 40-, 50-, 60-$, and 70-day-old (i.e. spanning pubertal and adolescent development) male rats before and after a $30 \mathrm{~min}$ session of restraint stress. We found that the adult-like ACTH stress response develops between 50 and 60 days of age, while
\end{abstract}

the corticosterone response changes between 30 and 40 days of age. We also found that adrenal corticosterone concentrations paralleled the plasma corticosterone response following restraint, suggesting that stress-induced adrenal corticosterone synthesis decreases during adolescent development and may, at least in part, contribute to the differential stress response observed before and after puberty. Finally, stress leads to increases in testosterone secretion, but only after 50 days of age. Collectively, these results indicate that shifts in hormonal stress responses occur throughout adolescent maturation and that these responses show distinct developmental profiles.

Journal of Endocrinology (2011) 210, 391-398

\section{Introduction}

Shifts in neuroendocrine function are a hallmark of pubertal and adolescent development (Sisk \& Foster 2004, Spear 2010). These changes allow an organism to successfully navigate the transition from a state of dependence on caregivers to a state of relative independence and sexual maturity (Spear 2010). Though puberty and adolescence have some chronological overlap, puberty is a relatively discrete physiological event, signaling the onset of adolescence, associated with dramatic hormonal and somatic changes, while adolescence is a more general term used to indicate the more protracted transitional period between childhood and adulthood. Though the exact age span that covers these developmental stages in the rat is not precisely defined, animals between 30 and 70 days of age demonstrate the greatest changes in somatic, behavioral, physiological, and neurobiological parameters during the juvenile to adult transition, akin to those demonstrated by human and non-human primates (Ojeda \& Urbanski 1994, Spear 2000, 2010, Sisk \& Foster 2004). Thus, basic animal models, such as the rat, provided an opportunity to study many of the fundamental physiological processes associated with these key life stages in an individual's development.

Recent research has drawn attention to the fact that pubertal and adolescent maturation in human and

non-human animals is marked by changes in hormonal stress responsiveness that may have profound consequences for an organism's short- and long-term physiological and neurobehavioral function (reviewed in McCormick \& Mathews (2007), Dahl \& Gunnar (2009), McCormick (2010), and Romeo $(2010 a, b))$. These pubertal shifts in stress reactivity can be quite striking. For instance, following an acute physical or psychological stressor, prepubertal rats exhibit an ACTH and corticosterone (total and free) response that is twice as long (e.g. 40-60 $\mathrm{min}$ ) as that exhibited by adults (Goldman et al. 1973, Vazquez \& Akil 1993, Romeo et al. 2004a,b, 2006a,b, Doremus-Fitzwater et al. 2009). Despite being a critical transition in hypothalamic-pituitaryadrenal (HPA) axis development (McCormick \& Mathews 2007, McCormick 2010, Romeo 2010a,b), the maturation of the hormonal stress response from the time of pubertal onset ( $\sim 30$ days of age) to adulthood ( $\sim 70$ days of age) has not been described. Moreover, it remains unclear what factors contribute to these changes in stress-induced hormone responses during pubertal and adolescent development.

In addition to pubertal-related shifts in ACTH and corticosterone secretion following stressors, prepubertal, and adult rats also display differential stress-induced changes in circulating gonadal hormone levels. Specifically, adult male rats show transient increases in plasma testosterone 
concentrations after exposure to a brief stressor (Siegel et al. 1981, Amariro \& Castellanos 1984, Romeo et al. 2004a), while prepubertal males show either no change or a suppression of testosterone secretion (Gomez et al. 2002, Romeo et al. 2004a). It is also important to note that in adulthood, gonadal hormones can modulate HPA reactivity (Handa et al. 1994, Viau \& Meaney 1996, Viau 2002), but that the gonadal hormones do not appear to mediate the prolonged stress responses observed in prepubertal animals (Romeo et al. 2004a,b).

Studying the adolescent development of the hormonal stress response will not only contribute to our basic understanding of how this crucial life stage affects the functioning of key neuroendocrine and physiological systems but may also shed light on the stress-related vulnerabilities associated with puberty and adolescence (Spear 2000, Andersen 2003, Costello et al. 2003, Dahl 2004, Patton \& Viner 2007). In this context, our present set of experiments had three objectives. First, we measured stress-induced $\mathrm{ACTH}$ and corticosterone responses in rats throughout pubertal and adolescent development to determine when the transformation to an adult-like pattern of hormonal stress reactivity occurs. Secondly, in an effort to evaluate the contribution of the adrenal glands to these remarkable pubertal changes in hormonal stress reactivity, we assessed adrenal corticosterone concentrations in response to both pubertal development and stress. Finally, we investigated whether mid-adolescent and adult males show similar or different testosterone secretion patterns following exposure to an acute stressor. Our overarching hypothesis is that there will be gradual transitions in these stress-induced hormonal responses throughout adolescent development to the more mature and adult-like response.

\section{Materials and Methods}

\section{Animals and housing}

Male Sprague-Dawley rats $(n=110)$ were obtained from Charles River (Wilmington, MA, USA) at 21 days of age, housed two per cage in clear polycarbonate cages $(45 \times 25 \times 20 \mathrm{~cm})$ with wood chip bedding, and maintained on a $12 \mathrm{~h}$ light: $12 \mathrm{~h}$ darkness schedule (lights on at $0900 \mathrm{~h}$ ). All animals had access to food and water ad libitum, and the animal room was maintained at $21 \pm 2{ }^{\circ} \mathrm{C}$. All procedures were carried out in accordance with the guidelines established by the National Institutes of Health Guide for the Care and Use of Laboratory Animals and approved by the Institutional Animal Care and Use Committee (IACUC) of Columbia University.

\section{Experimental design}

To examine pubertal and adolescent changes in stressinduced hormonal responses, plasma $\mathrm{ACTH}$, corticosterone, and testosterone levels, as well as adrenal corticosterone concentrations were measured in male rats at 30, 40, 50, 60, and 70 days of age. These ages approximately represent animals prior to, during, and after pubertal and adolescent maturation.

Animals were weighed and rapidly decapitated by a guillotine before (i.e. time 0) or after a $30 \mathrm{~min}$ session of restraint stress. Three time points following the restraint were examined: immediately (i.e. time 30) or 60 or $90 \mathrm{~min}$ after termination of the restraint ( $n=4-6$ per age and time point). Restraint was administered by placing animals in the prone position in wire mesh restrainers, sized so that animals at these different developmental stages were equally restrained. Animals in the 60 and $90 \mathrm{~min}$ groups were returned to their home cages until tissues were collected at the appropriate post-stress time point. All animals were killed between 1100 and $1300 \mathrm{~h}$ during their circadian nadir of ACTH and corticosterone release to minimize basal variations in these hormone levels (Romeo et al. 2006b). Blood, pituitary, adrenals, and testes were obtained from each animal. Trunk blood samples were collected in Vacutainer K3 EDTA-coated tubes (Fisher Scientific, Pittsburgh, PA, USA), spun down at $4{ }^{\circ} \mathrm{C}$ in a refrigerated centrifuge, plasma removed, and stored at $-20{ }^{\circ} \mathrm{C}$ until the RIAs were performed (see below). Adrenal glands were rapidly removed, cleaned of fat, snap frozen on powdered dry ice, and stored at $-80{ }^{\circ} \mathrm{C}$ until they were prepared for determination of protein and corticosterone concentrations (see below). Testes were removed, cleaned of fat, and weighed to verify developmental stage.

\section{RIA}

ACTH, corticosterone, and testosterone assays were conducted using commercially available kits, and reagents were performed as indicated by the supplier. ACTH levels were determined with an ACTH ${ }^{125}$ I Kit (DiaSorin, Inc., Stillwater, MN, USA) using the option A (overnight incubation) protocol. Corticosterone and testosterone levels were determined with Coat-A-Count ${ }^{125}$ I RIA Kits (Siemens Medical Solutions Diagnostics, Malvern, PA, USA). For each assay, all samples were run in duplicate. The lower limits of detectability and intraassay coefficient of variations for each assay were as follows: ACTH, $6.02 \mathrm{pg} / \mathrm{ml}$ and $14.8 \%$; corticosterone (plasma), $9.98 \mathrm{ng} / \mathrm{ml}$ and $7 \cdot 4 \%$ and (adrenal) $10.66 \mathrm{ng} / \mathrm{ml}$ and $7 \cdot 0 \%$; and testosterone, $0 \cdot 1 \mathrm{ng} / \mathrm{ml}$ and $6 \cdot 4 \%$.

\section{Determination of adrenal corticosterone concentration}

Adrenal protein and corticosterone concentrations were measured as described previously with slight modifications (Akana et al. 1992, Ulrich-Lai \& Engeland 2002, Figueiredo et al. 2007). Briefly, adrenal glands were weighed, homogenized in $20 \%$ ethanol in $0.9 \%$ saline, and centrifuged at $4000 \mathrm{~g}$ for $20 \mathrm{~min}$. The supernatants were diluted 1:5 in the homogenization buffer and stored at $-80^{\circ} \mathrm{C}$ for later 
Table 1 Mean ( \pm S.E.M.) body, paired testis, pituitary, and adrenal weights of male rats at 30, 40, 50, 60, and 70 days of age (days)

\begin{tabular}{|c|c|c|c|c|}
\hline Age (days) & Body weight (g) & Testes (g) & Pituitary (mg) & Adrenal (mg) \\
\hline 30 & $109 \cdot 58 \pm 1 \cdot 97^{\mathrm{a}}$ & $0 \cdot 88 \pm 0 \cdot 05^{a}$ & $7 \cdot 38 \pm 0 \cdot 08^{a}$ & $26 \cdot 12 \pm 0 \cdot 16^{a}$ \\
\hline 40 & $202 \cdot 67 \pm 4 \cdot 05^{\mathrm{b}}$ & $1 \cdot 69 \pm 0.04^{b}$ & $9 \cdot 65 \pm 0.09^{b}$ & $37 \cdot 32 \pm 0.02^{b}$ \\
\hline 50 & $294 \cdot 24 \pm 4 \cdot 68^{\mathrm{C}}$ & $2 \cdot 53 \pm 0.04^{c}$ & $12 \cdot 75 \pm 0 \cdot 16^{\mathrm{c}}$ & $47 \cdot 42 \pm 0 \cdot 38^{\mathrm{C}}$ \\
\hline 60 & $362 \cdot 15 \pm 6 \cdot 88^{d}$ & $2 \cdot 88 \pm 0 \cdot 07^{d}$ & $13 \cdot 83 \pm 0 \cdot 08^{\mathrm{c}}$ & $50 \cdot 15 \pm 0 \cdot 28^{\mathrm{C}}$ \\
\hline 70 & $422 \cdot 71 \pm 5.73^{\mathrm{e}}$ & $3 \cdot 15 \pm 0.03^{\mathrm{e}}$ & $14 \cdot 35 \pm 0 \cdot 10^{\mathrm{C}}$ & $57.07 \pm 0.67^{\mathrm{c}}$ \\
\hline
\end{tabular}

Note that the data are collapsed across stress time points. Values within a measure that share a letter are not significantly different from each other. Values with different letters are significantly different at $P<0 \cdot 05$.

measurement of protein and corticosterone. The protein concentrations were determined by the bicinchonicic acid method (Pierce, Rockford, IL, USA), while corticosterone levels were measured via RIA as described earlier. Adrenal corticosterone concentrations are expressed as nanogram corticosterone per milligram protein. These levels represent non-corticosteroid-binding globulin (CBG)-bound corticosterone as CBG expression is minimal in the adrenal gland (Scrocchi et al. 1993).

\section{Statistical analyses}

All data are presented as the mean \pm s.E.M. One- and two-way (age X time point) ANOVA were used for statistical analyses, while significant main effects and interactions were further analyzed with Tukey's honestly significant difference tests. Linear regression analyses were also conducted on the relationship between plasma corticosterone and ACTH levels at select ages and time points. Differences were considered significant when $P<0 \cdot 05$. All statistical analyses were performed using the GraphPad Prism Software (version 5.04, La Jolla, CA, USA).

\section{Results}

Peripheral indexes of pubertal and adolescent development

As expected, there were significant main effects of age on body, paired testis, pituitary, and adrenal weights $(\mathrm{F}(4,78)=558 \cdot 2$, $422 \cdot 4,46 \cdot 2$, and $66 \cdot 0$, respectively, $P<0 \cdot 05$, Table 1$)$. More specifically, body and paired testis weights increased significantly at each of the five ages tested, while pituitary and adrenal weights increased significantly between 30 and 50 days of age, after which their weights increased, but at a slower rate (Table 1). These data confirm that male rats between 30 and 70 days of age are indeed at disparate stages of pubertal and adolescent development.

\section{Plasma ACTH and corticosterone concentrations}

There was a significant interaction of age and time on plasma ACTH levels $(F(12,78)=6 \cdot 44, P<0 \cdot 05)$. In particular, though animals at all ages showed significantly elevated ACTH levels immediately following termination of the stressor (i.e. $30 \mathrm{~min}$ time point), this post-stress increase in ACTH was highest in animals at 30, 40, and 50 days of age (Fig. 1A). There were no other significant differences between the ages in plasma ACTH levels at the 0,60 , or 90 min time points.

For plasma corticosterone, there was also a significant interaction of age and time $(F(12,78)=8 \cdot 90, P<0 \cdot 05)$. Similar to the ACTH data, animals at all ages displayed
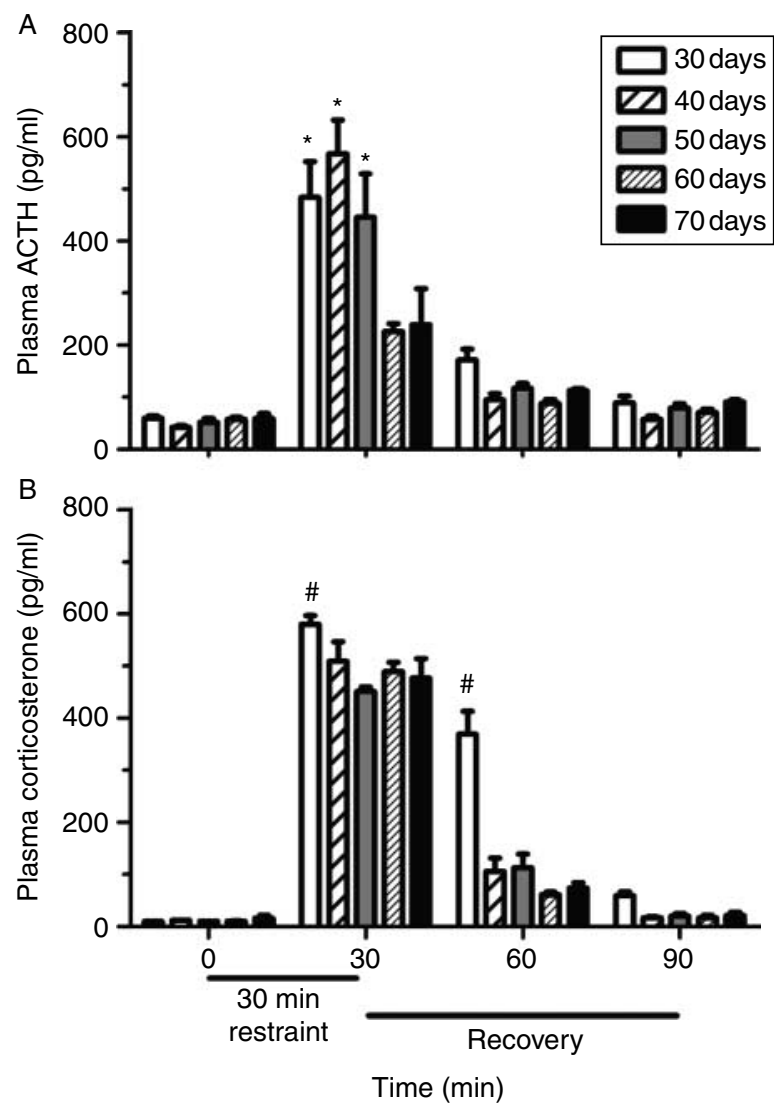

Figure 1 Mean ( \pm S.E.M.) plasma ACTH (A) and corticosterone (B) concentrations in 30-, 40-, 50-, 60-, and 70-day-old male rats before or after a $30 \mathrm{~min}$ session of restraint stress $(n=4-6$ per age and time point). *Indicate a significant difference from the $60-$ and 70-day-old animals at that time point, while ${ }^{\#}$ indicates that 30-day-old animals are significantly different from all other ages at that time point $(P<0 \cdot 05)$. 


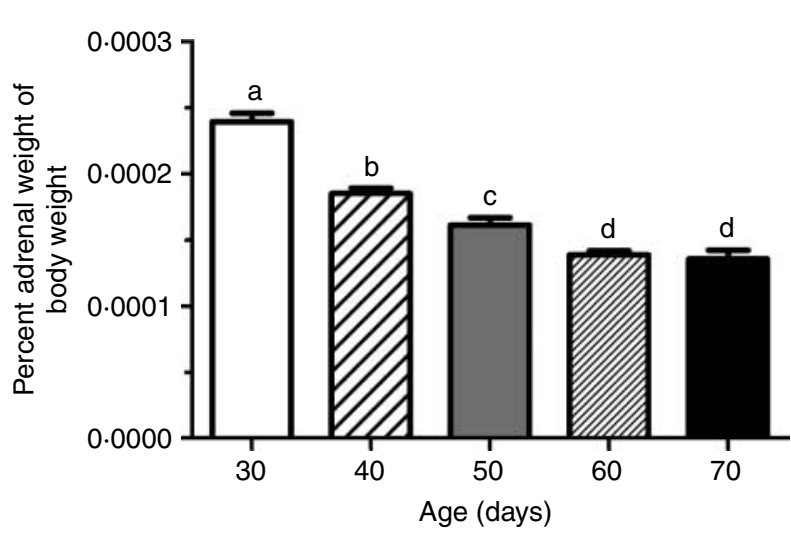

Figure 2 Mean ( \pm S.E.M.) percent adrenal weight of body weight in male rats at 30,40,50,60, and 70 days of age. Note that the data are collapsed across stress time points. Bars that share a letter are not significantly different from each other. Bars with different letters are significantly different at $P<0 \cdot 05$.

significant increases in corticosterone levels immediately following the stressor. However, 30-day-old animals had the highest stress-induced corticosterone levels at the $30 \mathrm{~min}$ time point and continued to demonstrate significantly higher levels at the 60 min time point compared with animals at all other ages (Fig. 1B). Despite these age-dependent effects at the 30 and 60 min time points, all animals returned to baseline 90 min after termination of the stressor.

As body and adrenal weights increase at different rates during these ages (see Table 1), we assessed whether a greater adrenal gland to body weight ratio (collapsed across stress time point) could in part account for the greater plasma corticosterone response in the 30-day-old animals. However, post hoc tests after the one-way ANOVA $(\mathrm{F}(4,97)=62 \cdot 77$, $P<0.05)$ revealed that the adrenal gland to body weight ratio significantly decreased in animals at each of the ages between 30 and 60 days of age (Fig. 2). Therefore, it appears that the differential plasma corticosterone response exhibited by the 30-day-old animals, compared to the animals between 40 and 70 days of age, cannot be attributed simply to differences in their adrenal gland to body weight ratio.

It appears from these plasma ACTH and corticosterone data that there may be age- and time-dependent shifts in adrenal responsiveness to ACTH. Specifically, it seems that during the earlier stages of the response (i.e. 0-30 $\mathrm{min}$ ), animals between 50 and 60 days of age show an increase in adrenal sensitivity, while later in the response (i.e. 60-90 $\mathrm{min}$ ), the 30-day-old animals appear to show a greater sensitivity. To further investigate this possible relationship, we conducted linear regression analyses between plasma ACTH and corticosterone levels in animals at these select ages and time points (Fig. 3A and B). As expected, there were significant positive correlations between the ACTH and corticosterone responses at these ages and time points (Fig. 3A; 50 days, $r^{2}=0.75 ; 60$ days, $r^{2}=0.97$ and Fig. 3B; 30 days, $r^{2}=0.76 ; 40$ days, $r^{2}=0.52$; all $P<0.05$ ) such that as ACTH levels rise corticosterone levels rise as well. More importantly, however, in the early portion of the response $(0-30 \mathrm{~min})$, the 60 -day regression line was significantly elevated compared to the 50-day line (Fig. 3A; $P<0 \cdot 05$ ), while in the later portion of the response $(60-90 \mathrm{~min})$, the 30 -day line was significantly elevated compared to the 40-day line (Fig. 3B; $P<0 \cdot 05$ ). The significantly different slopes of these regression lines indicate that there may be age- and time-dependent shifts in adrenal sensitivity to ACTH.

\section{Adrenal corticosterone concentrations}

We assessed adrenal corticosterone content in an effort to further investigate possible intermediate mediators of the differential stress-induced plasma corticosterone response in these animals. A two-way ANOVA revealed a significant interaction between age and time on adrenal corticosterone concentrations $(\mathrm{F}(12,78)=2 \cdot 44, P<0.05)$ such that stress exposure increased adrenal corticosterone levels across the ages, but that this elevation was significantly greater, and more protracted, in the 30-day-old animals compared with all other age groups (Fig. 4). Though there was a trend for 30-day-old animals to have higher basal levels of adrenal corticosterone, there were no significant differences between the ages at the 0 min time point.

\section{Plasma testosterone concentrations}

We also found age-dependent effects on the stress responsiveness of the gonadal axis in that we found a significant interaction between age and time on plasma testosterone concentration $(\mathrm{F}(12,78)=3 \cdot 68, P<0 \cdot 05)$. More specifically, at the basal time point, 40-, 50-, and 60-day-old males had higher testosterone levels compared with 30-day-old males, while 70-day-old males had significantly higher testosterone concentrations compared with all other age groups.
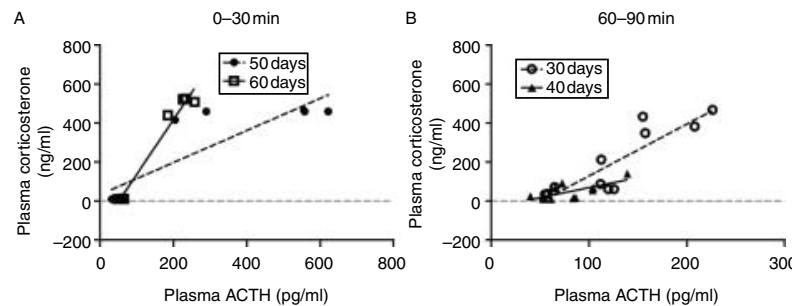

Figure 3 Correlational analyses of plasma corticosterone $(\mathrm{ng} / \mathrm{ml})$ and plasma ACTH $(\mathrm{pg} / \mathrm{ml})$ levels by re-plotting the individual data points from the plasma hormone analyses in Fig. 1. The relationship between the corticosterone and the ACTH response in 50- and 60-day-old animals from 0 to 30 min of stressor onset (A) and in 30and 40-day-old animals from 60 to 90 min of stressor onset (B). The regression lines in $\mathrm{A}$ (50 days, $r^{2}=0 \cdot 75 ; 60$ days, $\left.r^{2}=0.97\right)$ and $\mathrm{B}$ (30 days, $r^{2}=0 \cdot 76 ; 40$ days, $r^{2}=0 \cdot 52$ ) show a positive correlation. More importantly, however, in the early portion of the response (0-30 min; A), the 60-day regression line was significantly elevated compared to the 50-day line $(P<0 \cdot 05)$, while in the later portion of the response (60-90 min; B) the 30-day line was significantly elevated compared to the 40-day line $(P<0 \cdot 05)$. 


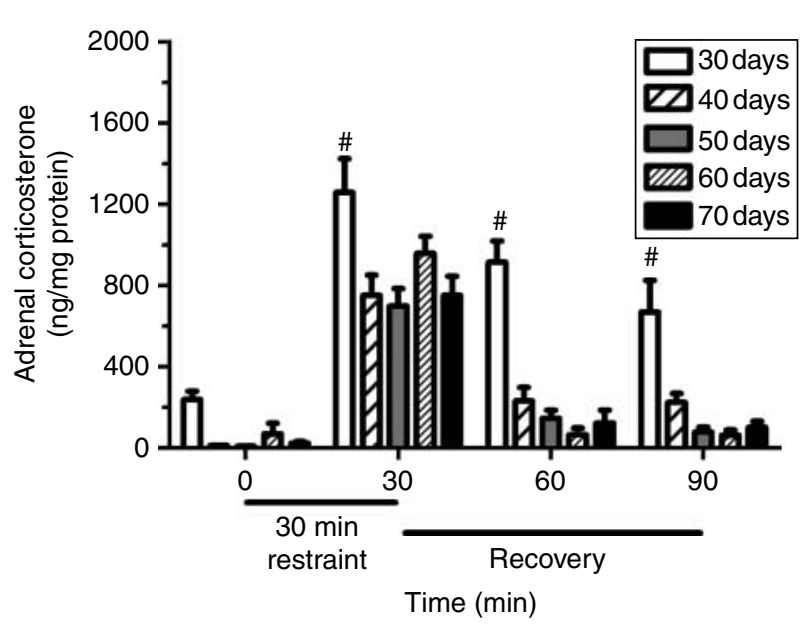

Figure 4 Mean ( \pm s.E.M.) adrenal corticosterone concentrations in 30-, 40-, 50-, 60-, and 70-day-old male rats before or after a $30 \mathrm{~min}$ session of restraint stress $\left(n=4-6\right.$ per age and time point). ${ }^{\#}$ Indicates that 30-day-old animals are significantly different from all other ages at that time point $(P<0 \cdot 05)$.

Furthermore, exposure to stress induced an increase in testosterone secretion in animals of 50,60, and 70 days of age, but not at 30 or 40 days of age. Post hoc tests also revealed that this stress-induced increase in testosterone was significant at all post-stress time points for animals at both 50 and 60 days of age. However, in 70-day-old animals, stress did not lead to increases in plasma testosterone levels until $1 \mathrm{~h}$ after termination of the stressor, at which time they significantly exceeded the levels of the 50- and 60-day-old animals (Fig. 5).

\section{Discussion}

These data clearly indicate pubertal and adolescent changes in the stress responsiveness of both the adrenal and the gonadal axes. Interestingly, in the context of the HPA axis, the age at which these changes occur depends on the node of the axis. For example, the shift in the stress-induced ACTH response occurs between 50 and 60 days of age, while the corticosterone response appears to mature earlier, between 30 and 40 days of age. Therefore, our results show that the stress responsiveness of the pituitary and adrenal glands may develop at different times or rates during this maturational stage. Moreover, the age-related changes in stress-induced testosterone secretion indicate that the effects of stress on other neuroendocrine axes, such as the HPG axis, are also dependent on pubertal development of the organism.

Numerous studies, using a variety of acute physical and/or psychological stressors such as foot shock, ether inhalation, and restraint, have indicated that prepubertal rats (25-32 days of age) display heightened ACTH and prolonged corticosterone responses compared with adults ( $>65$ days of age; Goldman et al. 1973, Vazquez \& Akil 1993, Romeo et al. 2004a,b, 2006a,b, Doremus-Fitzwater et al. 2009). Our present data replicate and extend these findings in that we report that the transformation to the adult-like ACTH and corticosterone response is relatively abrupt, occurring between 50 and 60 days of age and 30 and 40 days of age respectively.

Closer inspection of the $\mathrm{ACTH}$ and corticosterone responses also suggests shifts in adrenal sensitivity to ACTH that is both age and time dependent. Specifically, it appears that adult males (i.e. 60 and 70 days of age) display a greater adrenal sensitivity to ACTH during the earlier portions of the response (i.e. $0-30 \mathrm{~min}$ ) such that the ACTH response that was half that of the younger animals (i.e. 40 and 50 days of age) resulted in similar corticosterone responses across these ages. Conversely, $30 \mathrm{~min}$ after the stressor had been terminated, it appears that the prepubertal males (i.e. 30 days of age) show an increased adrenal sensitivity to ACTH in that ACTH levels are similar among the ages at the 60 min time point, but the prepubertal animals continue to mount a substantial corticosterone response. Though our regression analyses support this notion of age- and timedependent changes in adrenal responsiveness, future studies will need to directly assess these potential shifts in adrenal sensitivity by conducting ACTH dose-response curves in prepubertal and adult animals. Also, because our first blood samples were collected $30 \mathrm{~min}$ after the onset of the stressor, we are unable to determine any age-related differences in hormone secretion prior to this time point. As a previous study indicated that adult (65 days old) male rats demonstrate a greater corticosterone response than prepubertal (25 days old) males $15 \mathrm{~min}$ after the onset of a 3 min exposure to ether vapors (Vazquez \& Akil 1993), an experiment with sampling

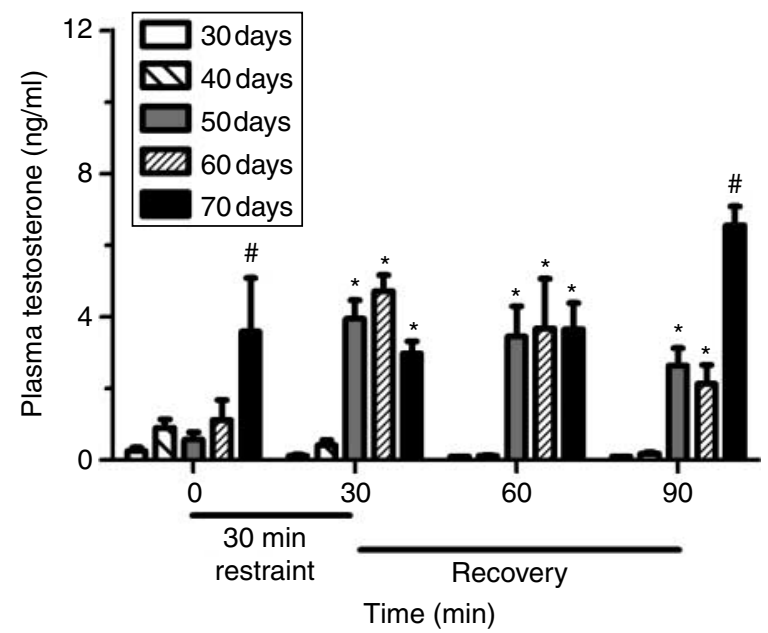

Figure 5 Mean ( \pm S.E.M.) plasma testosterone concentrations in 30-, $40-, 50-, 60-$, and 70 -day-old male rats before or after a $30 \mathrm{~min}$ session of restraint stress ( $n=4-6$ per age and time point). ${ }^{*}$ Indicates a significant difference from the 30- and 40-day-old animals at that time point, while ${ }^{\#}$ indicates that 70 -day-old animals are significantly different from all other ages at that time point $(P<0 \cdot 05)$. 
points during the $30 \mathrm{~min}$ session of restraint may help shed light on any age-dependent associations between ACTH and corticosterone secretion during the earlier phases of the HPA response.

Our current data are in accordance with an earlier study that demonstrated that 40-day-old male rats under conditions of longer periods of restraint stress (i.e. $3 \mathrm{~h}$ ) show a greater $\mathrm{ACTH}$ and slightly higher corticosterone response compared with 60-day-old males (Gomez et al. 2002). Thus, it appears that these age-dependent hormonal responses occur whether the duration of the acute stressor is short or relatively longer. It will also be important to establish whether these adolescent-related changes in stress reactivity generalize across different stress paradigms, as a recent report indicated that prepubertal animals actually show an abbreviated hormonal stress response compared to adults in reaction to an immune stressor (Goble et al. 2011).

Based on previous research on sex differences that suggested that the greater hormonal stress response demonstrated by adult female compared with adult male rats (Young 1998) may be, in part, mediated by the female's higher adrenal corticosterone concentrations (Chisari et al. 1995), we investigated whether differences in adrenal corticosterone may contribute to our age-related differences in stress reactivity. Indeed, our data show that following stress exposure, prepubertal animals have a higher adrenal corticosterone concentration than mid-pubertal and adult animals, and thus, the extended stress-induced corticosterone response prior to puberty may be related to this difference. Despite the fact that our adrenal homogenates contain the adrenal cortex and the medulla, the size of the adrenal cortex significantly increases in a fairly linear fashion between 30 and 70 days of age (Pignatelli et al. 2006), suggesting that our adrenal corticosterone data are not just an artifact of the corticosterone being diluted by growth restricted to the medulla. Regardless, future studies will need to assess changes in steroidogenic enzymes responsible for the production of corticosterone in the adrenal cortex, such as $11 \beta$-hydroxylase (Mellon et al. 1995, Engeland et al. 1997), as our data suggest, there may be a pubertal decline in these enzymes. Collectively, these data highlight the potential importance of the peripheral components of this neuroendocrine axis, such as the adrenal gland itself, in these age-related differences in responsiveness. It should be noted that other studies in adult rats have established the significance of higher adrenal corticosterone concentrations in the context of diurnal and chronic stress-induced changes in corticosterone secretion (Kaneko et al. 1981, Ulrich-Lai et al. 2006a,b).

Though our data indicate substantial changes in hormonal stress reactivity during puberty and adolescence, the mechanism(s) that mediate these changes are presently unclear. These differences do not appear to be due to altered metabolism of plasma corticosterone at these ages (Schaprio et al. 1971) nor based on changes in CBG levels (Romeo et al. 2006a). However, a previous study indicated that negative feedback on the prepubertal HPA axis following a stressor may be less than that on the adult (Goldman et al. 1973), while others have shown greater post-stress activation of the paraventricular nucleus of the hypothalamus, the brain region integral in initiating this hormonal cascade, and in prepubertal compared to adult rats (Viau et al. 2005, Romeo et al. 2006a). Thus, these reports point to at least a partial contribution of the central nervous system to these age-dependent changes in hormonal stress reactivity.

In addition to the differential ACTH and corticosterone responses, we found differences in stress-induced testosterone secretion between the ages; 50 days of age stress leads to an increase in testosterone secretion, while prior to 40 days it appears to suppress testosterone. Importantly, basal testosterone levels were similar in 40- and 50-day-old animals, so the lack of a post-stress testosterone 'surge' in the 40-day-old males cannot be solely attributed to the low levels of circulating testosterone at this age. Earlier studies in adult rats have indicated that acute stress leads to increases in $\mathrm{GnRH}$ (Lopez-Calderon et al. 1990) and LH (Euker et al. 1975, Lopez-Calderon et al. 1990) secretion, which likely mediate the stress-induced increases in testosterone we observe in the more sexually mature males in this study (i.e. $>50$ days of age). Though it is currently unknown what mediates the lack of a stress effect on testosterone secretion in our relatively immature animals (i.e. $<40$ days of age), perhaps the greater testosterone-mediated negative feedback on the prepubertal HPG (Negro-Vilar et al. 1973) causes any transient stressinduced increase in testosterone secretion in younger animals to rapidly inhibit its further release. The factors responsible for the delayed testosterone response in 70-day-old animals are also unknown, but this pattern of testosterone release may aid in preserving the reproductive capacity of older adults under stressful conditions (Gomez et al. 2002).

In conclusion, our results indicate that there are substantial shifts in hormonal stress reactivity throughout the adolescent stage of maturation and that the stress responsiveness of the pituitary, adrenals, and testes occur at different times during adolescence. With regard to the HPA axis, we found that the change in the ACTH response occurs between 50 and 60 days of age, while the corticosterone response transitions to its adult-like pattern between 30 and 40 days of age. As adrenal corticosterone concentrations parallel the stress-induced plasma corticosterone response, our data further suggest that the steroidogenic capacity of the adrenal gland may, at least in part, contribute to the differential stress response observed before and after puberty. Finally, the agerelated changes in testosterone secretion following an acute stressor highlight the fact that stress affects each neuroendocrine axis in distinct ways during the transition from puberty to adulthood.

\section{Declaration of interest}

The authors declare that there is no conflict of interest that could be perceived as prejudicing the impartiality of the research reported. 


\section{Funding}

This work was supported in part from a grant to Barnard College from the Undergraduate Science Education Program of the Howard Hughes Medical Institute and the National Science Foundation IOS-1022148 to R D R.

\section{Acknowledgements}

We would like to thank Page Buchanan for excellent animal care.

\section{References}

Akana SF, Scribner KA, Bradbury MJ, Strack AM, Dominique-Walker C \& Dallman MF 1992 Feedback sensitivity of the rat hypothalamo-pituitaryadrenal axis and its capacity to adjust to exogenous corticosterone. Endocrinology 131 585-594. (doi:10.1210/en.131.2.585)

Amariro A \& Castellanos JM 1984 Effect of acute and chronic stress on testosterone secretion in male rats. Journal of Endocrinological Investigation 7 659-661.

Andersen SL 2003 Trajectories of brain development: point of vulnerability or window of opportunity. Neuroscience and Biobehavioral Reviews 27 3-18. (doi:10.1016/S0149-7634(03)00005-8)

Chisari A, Carino M, Perone M, Gaillard RC \& Spinedi E 1995 Sex and strain variability in the rat hypothalamus-pituitary-adrenal (HPA) axis function. Journal of Endocrinological Investigation 18 25-33.

Costello EJ, Mustillo S, Erkanli A, Keeler G \& Angold A 2003 Prevalence and development of psychiatric disorders in childhood and adolescence. Archives of General Psychiatry 60 837-844. (doi:10.1001/archpsyc.60.8.837)

Dahl RE 2004 Adolescent brain development: a period of vulnerabilities and opportunities. Annals of the New York Academy of Sciences 1021 1-22. (doi:10.1196/annals.1308.001)

Dahl RE \& Gunnar MR 2009 Heightened stress responsiveness and emotional reactivity during pubertal maturation: implications for psychopathology. Development and Psychopathology 21 1-6. (doi:10.1017/ S0954579409000017)

Doremus-Fitzwater TL, Varlinskaya EI \& Spear LP 2009 Social and non-social anxiety in adolescent and adult rats after repeated restraint. Physiology $\&$ Behavior 97 484-494. (doi:10.1016/j.physbeh.2009.03.025)

Engeland WC, Levay-Young BK, Rogers LM \& Fitzgerald D 1997 Differential gene expression of cytochrome P450 11b-hydroxylase in rat adrenal cortex after in vivo activation. Endocrinology 138 2338-2346. (doi:10.1210/en.138.6.2338)

Euker JS, Meites J \& Riegle GD 1975 Effects of acute stress on serum LH and prolactin in intact, castrate and dexamethasone-treated male rats. Endocrinology 96 85-92. (doi:10.1210/endo-96-1-85)

Figueiredo HF, Ulrigh-Lai YM, Choi DC \& Herman JP 2007 Estrogen potentiates adrenocortical responses to stress in female rats. American Journal of Physiology 292 E1173-E1182. (doi:10.1152/ajpendo.00102.2006)

Goble KH, Bain ZA, Padow VA, Lui P, Klein ZA \& Romeo RD 2011 Pubertal-related changes in hypothalamic-pituitary-adrenal axis reactivity and cytokine secretion in response to an immunological stressor. Journal of Neuroendocrinology 23 129-135. (doi:10.1111/j.1365-2826.2010.02085.x)

Goldman L, Winget C, Hollingshead GW \& Levine S 1973 Postweaning development of negative feedback in the pituitary-adrenal system of the rat. Neuroendocrinology 12 199-211. (doi:10.1159/000122169)

Gomez F, Houshyar H \& Dallman MF 2002 Marked regulatory shifts in gonadal, adrenal, and metabolic system responses to repeated restraint stress occur within a 3-week period in prepubertal male rats. Endocrinology 143 2852-2862. (doi:10.1210/en.143.8.2852)

Handa RJ, Nunley KM, Lorens SA, Louie JP, McGivern RF \& Bollnow MR 1994 Androgen regulation of adrenocorticotropin and corticosterone secretion in the male rat following novelty and foot shock stressors. Physiology \& Behavior 55 117-124. (doi:10.1016/0031-9384(94)90018-3)
Kaneko M, Kaneko K, Shinsako J \& Dallman MF 1981 Adrenal sensitivity to adrenocorticotropin varies diurnally. Endocrinology 109 70-75. (doi:10. 1210/endo-109-1-70)

Lopez-Calderon A, Gonzalez-Quijano MI, Tresguerres JAF \& Ariznavarreta C 1990 Role of LHRH in the gonadotrophin response to restraint stress in intact male rats. Journal of Endocrinology 124 241-246. (doi:10.1677/joe.0. 1240241)

McCormick CM 2010 An animal model of social instability stress in adolescence and risk for drugs of abuse. Physiology \& Behavior 99 194-203. (doi:10.1016/j.physbeh.2009.01.014)

McCormick CM \& Mathews IZ 2007 HPA function in adolescence: role of sex hormones in its regulation and the enduring consequences of exposure to stressors. Pharmacology, Biochemistry, and Behavior 86 220-233. (doi:10. 1016/j.pbb.2006.07.012)

Mellon SH, Bair SR \& Monis H 1995 P450c11B3 mRNA, transcribed from a third P450c11 gene, is expressed in a tissue-specific, developmentally, and hormonally regulated fashion in the rodent adrenal and encodes a protein with both 11-hydroxylase and 18-hydroxylase activity. Journal of Biological Chemistry 270 1643-1649. (doi:10.1074/jbc.270.4.1643)

Negro-Vilar A, Ojeda SR \& McCann SM 1973 Evidence for changes in sensitivity to testosterone negative feedback on gonadatropin release during sexual development in the male rat. Endocrinology 93 729-735. (doi:10.1210/endo-93-3-729)

Ojeda SR \& Urbanski HF 1994 Puberty in the rat. In The Physiology of Reproduction, pp 363-409. Eds E Knobil \& JD Neill. New York: Raven Press.

Patton GC \& Viner R 2007 Pubertal transitions in health. Lancet 369 1130-1139. (doi:10.1016/S0140-6736(07)60366-3)

Pignatelli D, Xiao F, Gouveia AM, Ferreria JG \& Vinson GP 2006 Adrenarche in the rat. Journal of Endocrinology 191 301-308. (doi:10.1677/joe.1.06972)

Romeo RD 2010a Adolescence: a central event in shaping stress reactivity. Developmental Psychobiology 52 244-253. (doi:10.1002/dev.20437)

Romeo RD $2010 b$ Pubertal maturation and programming of hypothalamicpituitary-adrenal reactivity. Frontiers in Neuroendocrinology 31 232-240. (doi:10.1016/j.yfrne.2010.02.004)

Romeo RD, Lee SJ, Chhua N, McPherson CR \& McEwen BS 2004a Testosterone cannot activate an adult-like stress response in prepubertal male rats. Neuroendocrinology 79 125-132. (doi:10.1159/000077270)

Romeo RD, Lee SJ \& McEwen BS 2004b Differential stress reactivity in intact and ovariectomized prepubertal and adult female rats. Neuroendocrinology $\mathbf{8 0}$ 387-393. (doi:10.1159/000084203)

Romeo RD, Bellani R, Karatsoreos IN, Chhua N, Vernov M, Conrad CD \& McEwen BS 2006a Stress history and pubertal development interact to shape hypothalamic-pituitary-adrenal axis plasticity. Endocrinology 147 1664-1674. (doi:10.1210/en.2005-1432)

Romeo RD, Karatsoreos IN \& McEwen BS $2006 b$ Pubertal maturation and time of day differentially affect behavioral and neuroendocrine responses following an acute stressor. Hormones and Behavior 50 463-468. (doi:10. 1016/j.yhbeh.2006.06.002)

Schaprio S, Percin CJ \& Kotichas FJ 1971 Half-life of plasma corticosterone during development. Endocrinology 89 284-286. (doi:10.1210/ endo-89-1-284)

Scrocchi LA, Orava M, Smith CL, Han VKM \& Hammond GL 1993 Spatial and temporal distribution of corticosteroid-binding globulin and its messenger ribonucleic acid in embryonic and fetal mice. Endocrinology $\mathbf{1 3 2}$ 903-909. (doi:10.1210/en.132.2.903)

Siegel RA, Weindenfeld J, Feldon S, Conforti N \& Chowers I 1981 Neural pathways mediating basal and stress-induced secretion of luteinizing hormone, follicle-stimulating hormone, and testosterone in the rat. Endocrinology 108 2302-2307. (doi:10.1210/endo-108-6-2302)

Sisk CL \& Foster DL 2004 The neural basis of puberty and adolescence. Nature Neuroscience 7 1040-1047. (doi:10.1038/nn1326)

Spear LP 2000 The adolescent brain and age-related behavioral manifestations. Neuroscience and Biobehavioral Reviews 24 417-463. (doi:10.1016/ S0149-7634(00)00014-2)

Spear LP 2010 Sex differences, puberty, and the hormonal reawakening of adolescence (chapter 3). In The Behavioral Neuroscience of Adolescence, p 36. New York: Norton. 
Ulrich-Lai YM \& Engeland WC 2002 Adrenal splanchnic innervation modulates adrenal cortical responses to dehydration stress. Neuroendocrinology 76 79-92. (doi:10.1159/000064426)

Ulrich-Lai YM, Arnhold MM \& Engeland WC 2006a Adrenal splanchnic innervation contributes to the diurnal rhythm of plasma corticosterone in rats by modulating adrenal sensitivity to ACTH. American Journal of Physiology 290 R1128-R1135. (doi:10.1152/ajpregu.00042.2003)

Ulrich-Lai YM, Figueiredo HF, Ostrander MM, Choi DC, Engeland WC \& Herman JP $2006 b$ Chronic stress induces adrenal hyperplasia and hypertrophy in a subregion-specific manner. American Journal of Physiology 291 E965-E973. (doi:10.1152/ajpendo.00070.2006)

Vazquez DM \& Akil H 1993 Pituitary-adrenal response to ether vapor in the weanling animal: characterization of the inhibitory effect of glucocorticoids on adrenocorticotropin secretion. Pediatric Research 34 646-653.

Viau V 2002 Functional cross-talk between the hypothalamic-pituitarygonadal and adrenal axes. Journal of Neuroendocrinology 14 506-513. (doi:10. 1046/j.1365-2826.2002.00798.x)
Viau V \& Meaney MJ 1996 The inhibitory effect of testosterone on hypothalamic-pituitary-adrenal responses to stress is mediated by the medial preoptic area. Journal of Neuroscience 16 1866-1876.

Viau V, Bingham B, Davis J, Lee P \& Wong M 2005 Gender and puberty interact on the stress-induced activation of parvocellular neurosecretory neurons and corticotropin-releasing hormone messenger ribonucleic acid expression in the rat. Endocrinology 146 137-146. (doi:10.1210/ en.2004-0846)

Young EA 1998 Sex differences and the HPA axis: implications for psychiatric disease. Journal of Gender-Specific Medicine 1 21-27.

Received in final form 5 July 2011

Accepted 11 July 2011

Made available online as an Accepted Preprint

11 July 2011 